\title{
Virtual Ruler: Mobile Beacon Based Distance Measurements for Indoor Sensor Localization
}

\author{
Chen Wang, Yong Ding, Li Xiao \\ Department of Computer Science and Engineering \\ Michigan State University \\ Email: $\{$ wangchen, dingyong, lxiao\}@cse.msu.edu
}

\begin{abstract}
In sensor localization, ultrasound based distance measurements will have large errors if line-of-sight paths are blocked between pairwise sensors. Because these outliers, once mixed together with other correct distance measurements, are difficult for localization algorithms to identify, we propose to exclude the outliers in the first step of distance measurement. Although the distance between pairwise sensors measured by ultrasound can have multiple values due to the multipath effect, our experiments find that a sensor's incorrect position, estimated from the distance measurement along a reflected path instead of a straight line, is always mirrored to the sensor's correct position. Based on this phenomena, we propose to use mobile beacons to measure the distance between pairwise sensors from multiple perspectives and filter incorrect values through a statistical approach. Our performance evaluation shows that the proposed algorithm can achieve better localization results than previous approaches in an indoor environment where multipath effects cannot be avoided.
\end{abstract}

\section{INTRODUCTION}

Numerous applications [1][2][3] and protocols [4][5] in sensor networks necessitate sensors to locate themselves, which usually involves two steps: (i) in-network distance measurement between pairwise nodes and (ii) geometric calculation based on measured distances. For the first step, radio signals and ultrasound are widely used as the distance measurement media. Because radio signals attenuate during their transmission, the transmission distance can be estimated from the received radio signal strength (RSS). However, the distance estimated by the RSS approach is often unreliable and inaccurate because radio signals are susceptible to environmental interference. The ultrasound approach estimates the distance between pairwise sensors by multiplying the time of flight (ToF) with sound's constant speed. Accurate distance measurement can be achieved by the ultrasound approach if a line-of-sight path exists between pairwise sensors.
Despite its accurate results in the line-of-sight condition, the ultrasound ToF approach must address two challenges before it can be readily applied to a sensor network deployed in a complicated environment, especially in an indoor environment where ultrasound signals are reflected along multiple paths. The first challenge is that the ultrasound ToF approach has short measurable range due to the power constraint of sensor nodes. The second challenge is that distance measurements will have large errors in an obstructed environment. As shown in Fig. 1, when the line-of-sight path is blocked between sensor $S_{1}$ and $S_{2}$, the distance has to be estimated from a reflected path which is much longer than the true distance between $S_{1}$ and $S_{2}$. In this paper, we try to address these two challenges by using mobile beacons to measure distances between pairwise sensors deployed in an indoor environment.

To measure distance between pairwise sensors, we propose to use a pair of beacons attached to a small vehicle which randomly moves around in the deployed area. In our proposed approach, only beacons are equipped with ultrasound senders, and sensors are equipped with receivers that passively receive ultrasound signals broadcast by beacons. The distance between pairwise sensors can be determined if both sensors are within the beacons' ultrasound transmission range. When mobile beacons move around in the deployed area, it is possible to obtain sufficient distance constraints through which sensors' relative positions can be uniquely determined. Here, mobile beacons behave as a virtual ruler that wanders in the deployed area to provide distance measurement service to pairwise sensors as shown in Fig. 2. Compared with previous ultrasound based distance measurement approaches where a sensor acts as both a sender and a receiver, the virtual ruler approach can achieve longer distance measurement range such that more distance constraints are available to form a rigid network to uniquely determine sensors' positions. Such a long range 
distance measurement can be achieved without violation of the energy constraints, because only beacons are equipped with high power ultrasound senders and sensors are equipped with receivers consuming less energy. As a result the virtual ruler approach addresses the first challenge of short distance measurement range.

To address the second challenge that distances estimated along the reflected paths have large errors, we conduct intensive experiments to test ultrasound distance measurement in an indoor environment. We observe that a sensor's incorrect position, estimated from a reflected path instead of a straight line, is always mirrored to its true position. As shown in Fig. 1, the distance estimated between sensor $S_{1}$ and $S_{2}$ along the reflected path is equal to the distance between $S_{1}$ and $S_{2}$, the mirrored position of $S_{2}$. Based on this phenomena, we further observed that incorrect distance measurements incurred by multipath effect have finite values that are virtual distances between sensors and their mirrors. This makes it possible to identify incorrect distance measurements through a statistical approach. When the virtual ruler moves around a pair of sensors, the distance between the pair of sensors can be measured by the virtual ruler multiple times from different perspectives. We observe two phenomena: i) a distance between the same pairwise sensors can be measured by the virtual ruler more frequently if it is less affected by obstructions; ii) among all the measured values to the same distance, the correct measurement value is observed more frequently than incorrect ones. Therefore, we can identify correct distance measurements based on the distribution of measured values. Moreover, the confidence to a distance measurement can be quantified according to the distribution of its measured values. Based on the measurement confidence, the mobile distance measurement can be further combined with the recursive approach such that the distance measurement with higher confidence will have higher priority to be applied in the recursive approach.

The rest of the paper is organized as follows. Section 2 summarizes previous work. Section 3 describes the virtual ruler approach. Section 4 evaluates our proposed virtual ruler approach by comparing it with previous work. We conclude this paper in Section 5.

\section{RELATED WORK}

Because knowing sensors' positions is essential to many sensor applications, numerous approaches have been proposed to locate sensors with different tradeoff between accuracy and cost. The localization approaches can be broadly categorized as radio based approaches and ultrasound based approaches according to their innetwork distance measurement media.

Due to its unreliable and inaccurate nature, the radio based RSS approach can only give a coarse estimation of distances between pairwise sensors [6][7]. Instead of acquiring distance knowledge, radio signals are often used to estimate the connectivity between pairwise sensors, $\mathrm{i}$. e. whether a pair of sensors are within the maximum radio transmission range. Based on the connectivity information obtained from radio signals, the Centroid approach has been proposed to estimate a sensor's position as the centroid of the polygon formed by beacons that are connected to the estimated sensor through radio signals [8]. The accuracy of the Centroid approach has been further improved by the APIT approach that pinpoints the estimated sensor to a smaller area intersected by multiple triangles [9]. By approximating the hop count distance to the Euclidean distance between pairwise sensors, multihop approaches [10][11][12] were proposed to locate sensors in the case where beacons are sparsely distributed. Because sensors' positions estimated by radio based RSS approaches often have large errors, these approaches are suitable for applications that have less demand on localization accuracy.

Most indoor sensor applications require accurate localization results because sensors are deployed in a relatively small scale. In order for sensors to identify themselves based on their geographic coordinates, accurate localization results are necessary if they are densely deployed in a small area where distances between neighbors are short. Ultrasound based approaches, which are more reliable and accurate than radio based approaches, are well suited to the indoor cases [13]. By using ultrasound to accurately measure distances from sensors to nearby static beacons, sensors' positions can be determined with high accuracy. When beacons are sparsely distributed and not accessible to all sensors, the recursive approaches [14][15] have been proposed, which convert sensors to beacons after their positions have been determined. Thus, beacons can be propagated in a deployed area and accessed by all sensors. To eliminate errors, especially the large flipping errors, accumulated along the recursive approaches, the robust quad has been proposed to identify the situation where beacons are positioned closed to a straight line [16]. All above work focuses on the localization algorithm using static in-network distance measurement and assumes distance measurements have small errors that can be tolerated by localization algorithms. In contrast, our work investigates how to use mobile beacons to 


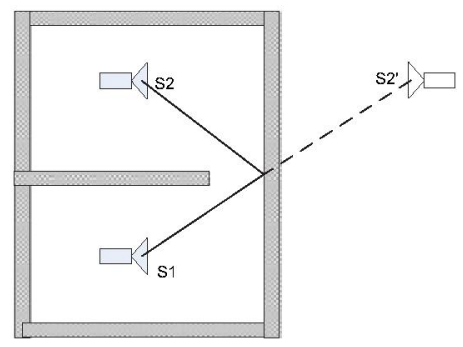

Fig. 1 Ultrasound multipath effect in an indoor environment

dynamically measure distances in an indoor environment and proposes to eliminate incorrect distances estimated along reflected paths in the measurement step.

Using mobile nodes in ad hoc wireless networks has been suggested by previous work [17][18]. Especially, using a mobile beacon to locate sensors has been proposed in [19], which assumes that the mobile beacon is equipped with GPS and know its absolute coordinate. When the mobile beacon moves around a sensor, the sensor can estimate the distances to various positions of the mobile beacon. Based on its relative distances to the mobile beacon, the sensor's absolute coordinate can be determined. Unlike this approach, our virtual ruler uses a pair of mobile beacons to measure distances between pairwise sensors instead of distances between sensors and beacons. The measured distances between pairwise sensors can be utilized by various localization algorithms to finally determine sensors' relative coordinates. The virtual ruler approach does not require GPS to determine absolute coordinates of beacons, which is attractive for indoor localization where GPS signals are difficult to receive.

The mobile-assisted approach proposed in [20] is similar to our work in that the beacons measure distances between pairwise nodes without reliance on GPS signals. Different from our work, the mobile-assisted approach uses a single mobile beacon to obtain distances between pairwise sensors. In order to have sufficient constraints to calculate the distance between pairwise sensors, the mobile-assisted approach requires the beacon to move along a certain track. For example, the mobile beacon must move along a straight line for two consecutive steps in the two dimensional localization. Moreover, the mobile-assisted approach pays less attention to the incorrect distance measurement incurred by obstructions.

Based on distances acquired by mobile beacons, the iterative least squares fitting is proposed in [21] to exclude an incorrect distance measurement in each iteration

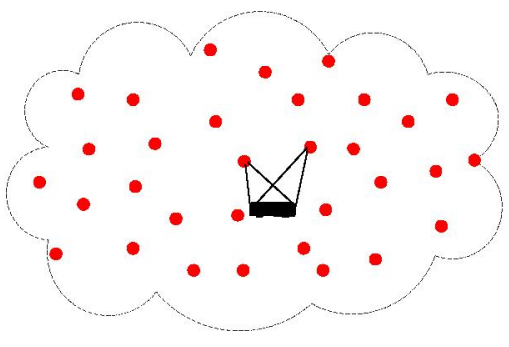

Fig. 2 The virtual ruler moves around in the deployed area

when a measured distance significantly differs from the estimated distance. In contrast, our virtual ruler approach proposes to exclude the incorrect distances in the measurement step instead of the localization algorithm. Detailed performance comparison between the virtual ruler approach, the mobile assisted approach, and the iterative least square fitting approach is conducted in Section 4

Methods of using ultrasound to measure distances between pairwise sensors have been extensively studied in [22][23]. Based on their work, we further investigate the ultrasound behaviors in an obstructed environment where multipath effect cannot be avoided.

\section{VirTUAL RULER DISTANCE MEASUREMENT APPROACH}

In this section, we first evaluate the measurement accuracy of the ultrasound ToF approach in the line-ofsight condition. After that, we test the ultrasound ToF approach in an obstructed environment. Based on our experimental observations, we propose the virtual ruler approach that can measure distances between pairwise sensors from multiple perspectives and filter incorrect distances statistically. How to combine the virtual ruler approach with the recursive approach is included at the end of this section.

\section{A. Ultrasound distance measurement in the line-of-sight condition}

Using ultrasound to measure distances in a sensor network has been extensively studied in previous work [13][23]. In this paper, we repeat the experiment of distance measurement in a line-of-sight condition and compare the result with that in an obstructed environment. We use the same hardware as the Cricket system [24], which attaches two ultrasound transceivers to the MICA2 nodes developed by UC Berkeley [25]. The Cricket sensor nodes broadcast the radio signals and ultrasonic signals at the same time. The ultrasonic signals 


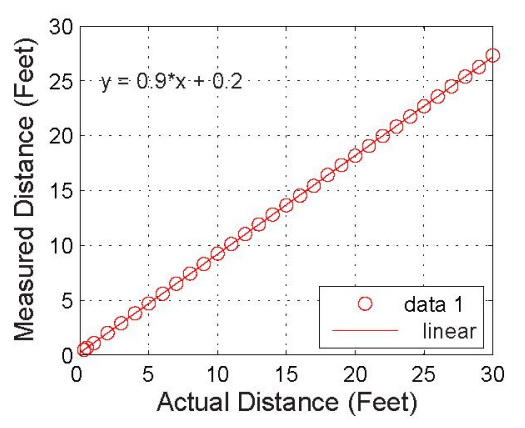

Fig. 3 Ultrasound calibration experiment

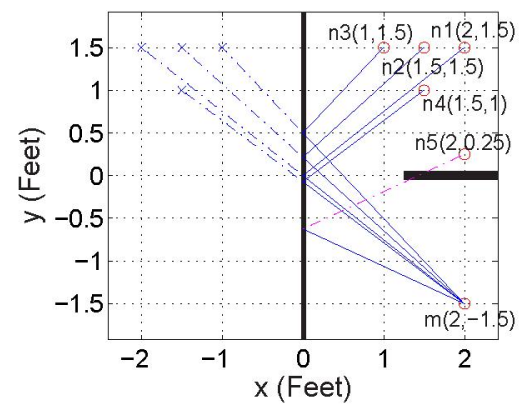

Fig. 4 Experiment of multipath effect will reach a receiving node later than the radio signals due to their speed difference. By measuring the flight delay of the ultrasonic signals, the distance between sender and receiver can be estimated based on the constant speed of ultrasonic signals.

We measure the distances between a pair of Cricket sensor nodes in the line-of-sight condition. The pair of sensors are placed on platforms 2 inches above a hallway's floor with ultrasound transceivers facing towards each other. We vary the distances between pairwise sensors from 0.3 feet to 30 feet. The experimental result is shown in Fig. 3, where $x$-axis represents the actual distances and $y$-axis represents the estimated distances. Fig. 3 shows that a clear linear relationship exists between the actual distances and estimated distances. The actual distances are slightly larger than the measured distances because sensors cannot be manufactured exactly the same as each other such that each sensor may use a slightly different time to process radio and ultrasound signals. However, the measurement errors caused by manufactory difference are a constant and can be easily calibrated through a fitting function between the actual distances and estimated distances. The fitting function for the pair of sensors used in this experiment is $y=0.9 \times x+0.2$. After calibration, the ultrasound approach can achieve high distance measurement accuracy. Methods for calibrating sensors in large volume has been studied in [23].

\section{B. Ultrasound distance measurement in an obstructed environment}

Despite its high measurement accuracy in the lineof-sight condition, the ultrasound approach may have large measurement errors in an indoor obstructed environment where multipath effects cannot be avoided. In an indoor environment, the ultrasonic signals may travel along multiple paths and arrive in a receiver at different time, though they start at the sender simultaneously. Nevertheless, if the line-of-sight path exists between pairwise sensors, the receiver can always identify the flight time along the shortest straight line by selecting the earliest arrival time of the ultrasonic signals. The situation becomes worse if a line-of-sight path does not exist between the sender and the receiver. As shown in Fig. 4, because the straight line between a sender and a receiver is blocked by an obstruction, the ultrasonic signals have to travel a longer distance along reflected paths. Consequently, the estimated distance is much longer than the Euclidean distance between the pairwise sensors.

Because the ultrasonic signals along the reflected paths have the same format as those along the straight lines, it is impossible for a single receiver to identify incorrect distance measurements affected by obstructions. However, our experiments show that a sensor's false position estimated by the reflected path is always mirrored to the sensor's true position. In other words, the distance estimated along the reflected path between pairwise sensors is exactly equal to the distance between the sender and the mirror of the receiver, which is shown in Fig. 4, where the true positions of the receivers are represented as circles and the mirrored positions are represented as crosses. We verify this phenomena through a series of experiments. As shown in Fig. 4, we put the sender in the fixed position $m$ while varying the receiver's position from $n_{1}$ to $n_{5}$. Because the line-ofsight path between the sender and the receiver is blocked by the obstruction, the ultrasonic signals have to travel along the path reflected by the wall. The distances of the reflected paths between the sender and various positions of the receivers are measured by the ultrasound, and we compare the measured distances with the actual distances between the sender and the mirrored positions of the receiver. 
TABLE I Comparison between the reflected distances and the distances from the sender to the receiver's mirrored positions

\begin{tabular}{|c|c|c|c|c|}
\hline positions & measured distance & calibrated distance & distance to mirrored position & Euclidean distance \\
\hline$m-n_{1}$ & 4.95 & 5.28 & 5.00 & 3.00 \\
\hline$m-n_{2}$ & 4.59 & 4.88 & 4.61 & 3.04 \\
\hline$m-n_{3}$ & 4.17 & 4.41 & 4.24 & 3.16 \\
\hline$m-n_{4}$ & 4.36 & 4.62 & 4.30 & 2.55 \\
\hline$m-n_{5}$ & $\mathrm{~N} / \mathrm{A}$ & $\mathrm{N} / \mathrm{A}$ & $\mathrm{N} / \mathrm{A}$ & 1.75 \\
\hline
\end{tabular}

These experiments are demonstrated in Fig. 4, where the solid lines are the reflected paths from the sender $m$ to the receiver's positions $n_{1}$ to $n_{4}$, while the dotted lines are the distances between the sender $m$ to the mirrored positions of $n_{1}$ to $n_{4}$. The receiver at position $n_{5}$ cannot receive any ultrasound signals from $m$, because even the signals reflected by the wall are blocked by the obstruction. Table I lists the comparison results between the measured distances along the reflected paths and the calculated distances between the sender and mirrored positions of the receiver. Table I shows that the measured distances of the reflected paths are much larger than the Euclidean distances between the sender and the receiver but close to the distances between the sender and mirrored positions of the receiver.

Because the incorrect distance measurement between pairwise sensors is always equal to the virtual distance from one sensor to the mirrored position of the other, we can conclude the incorrect distance measurements incurred by obstructions have finite values since the mirrored positions of a sensor are finite. This observation motivates us to measure the distance between pairwise sensors from multiple perspectives and filter out finite number of incorrect distance measurements through a statistical approach.

\section{Measure distances through the virtual ruler}

In order to measure distances between pairwise sensors from multiple perspectives, we attach two beacons to a small vehicle that randomly moves around in the deployed area. Because the distance between the attached beacons is fixed, we can easily infer the distance between a pair of sensors if the distances from the sensors to both beacons are known. Here, the mobile beacons behave as a virtual ruler that moves around to measure pairwise distances between sensors. Moreover, the distance between the same pair of sensors can be measured multiple times when mobile beacons move to different locations. Using mobile beacons to measure the distance between pairwise sensors is shown in Fig. 5, where node $m_{1}$ and $m_{2}$ are mobile beacons and node $n_{1}$ and $n_{2}$ are sensors. Because beacons' relative positions are fixed to each other, relative coordinates can be assigned to all the beacons. Here, we assign $m_{1}$ 's coordinate as $(0,0)$ and $m_{2}$ 's coordinate as $(0, L)$, where $L$ is the fixed length between $m_{1}$ and $m_{2}$. By measuring distances $d_{i j}$ from node $n_{i}$ to beacon $m_{j}$, the relative position of sensor $n_{i}$ can be calculated as below.

$$
\widehat{\mathbf{n}_{i}}=\arg \min _{\mathbf{n}_{i}} \sum\left(\left|\mathbf{n}_{i}-\mathbf{m}_{j}\right|-d_{i j}\right)^{2}
$$

Based on the relative position of $n_{1}$ and $n_{2}$, the Euclidean distance between the pairwise sensors can be simply calculated as $\left|\mathbf{n}_{i}-\mathbf{n}_{j}\right|$. We utilize the ultrasound's mono-directional transmission to avoid flip over error when two beacons are used. More robust result can be achieved when three beacons are laid out as a triangle. Intuitively, the error of the distance estimated by the virtual ruler is related to the fixed length $L$ between pairwise beacons. Higher distance measurement accuracy can be achieved by the longer length $L$. To evaluate the relationship between the distance measurement error and the length $L$, we conduct a series of experiments by varying the length $L$. The measurement error under different value of $L$ is shown in Fig. 6, which illustrates that sufficient measurement accuracy can be achieved with the relatively small length $L$. Consequently, we can implement the virtual ruler by attaching the beacons to a small vehicle.

Although high accuracy can be achieved by the virtual ruler when line-of-sight paths exist from pairwise sensors to both beacons, the distance measurement may have large errors in an obstructed environment where some of the distances from pairwise sensors to beacons are measured along reflected paths. However, as we discussed in Section III-B, the incorrect distance measurements caused by obstructions have finite values. We use the virtual ruler to measure the distance between a pair of sensors in a floor environment. The distribution of the measured value is shown in Fig. 7, where y-axis represents the measured values and x-axis represents the location from which the value is measured. Fig. 7 illustrates that only several values are obtained by the 


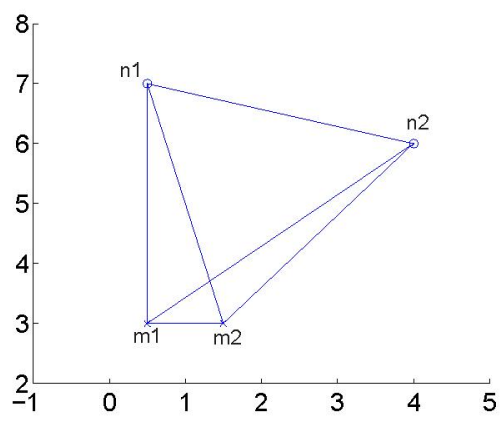

Fig. 5 Using mobile beacons to measure distance between pairwise sensors

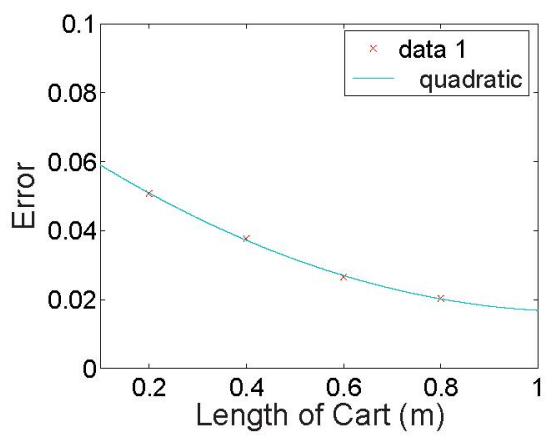

Fig. 6 Impact of fixed distance between mobile beacons on the measurement errors

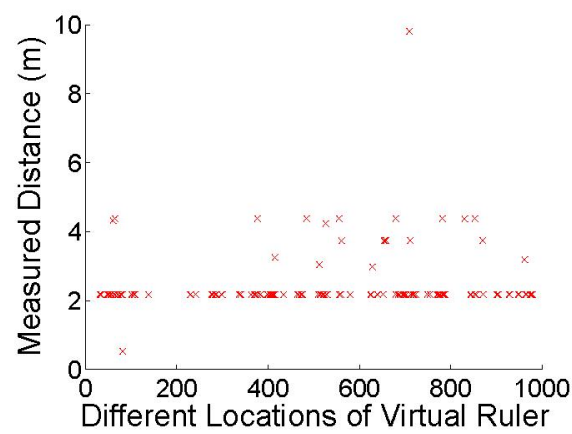

Fig. 7 Distance measurement value distribution obtained by mobile beacons virtual ruler. The distance measurement to the same pair of sensors has finite values because incorrect distances are equal to the virtual distances between a sensor to the finite mirrored positions of the other sensor. To further explain this phenomena, we list three examples in Fig. 8, where $m_{1}$ and $m_{2}$ represent the mobile beacons and $n_{1}$ and $n_{2}$ represent sensors, and the distance between $n_{1}$ and $n_{2}$ represents the incorrect distance estimation due to the multipath effects. Fig. 8(a), Fig. 8(b), and Fig. 8(c) show that 1,2 , and 4 line-of-sight paths are blocked by the obstruction respectively. An interesting observation is that when 4 line-of-sight paths are blocked by the obstruction in Fig. 8(c), both sensors are mirrored to the other side of the wall. As a result, the distance between the mirrored positions is equal to the distance between the original pairwise sensors. Therefore, the distance is correctly estimated by the mobile beacons, thought they measure distances along the reflected paths to the sensors.

When the virtual ruler moves around in the deployed area, it will measure distances between pairwise sensors after each moving step. During the movement, the virtual ruler can measure the distance between a pair of sensors from different perspectives to obtain different values, among which the correct distance measurement is mixed with the incorrect ones incurred by obstructions. In the following discussion, we show how to identify a correct distance measurement by assigning a confidence value to the measured distance.

\section{Evaluate the distances measured by the virtual ruler}

In order to identify correct distance measurements, we conduct intensive simulations in various obstructed environments. When the virtual ruler moves around a pair of sensors, it can observe different distance measurement values $d_{1}, d_{2}, \ldots d_{n}$ between the same pair of sensors from different perspectives. Moreover, the same value $d_{i}$ can be measured by the virtual ruler multiple times from different locations. Let $k_{i}$ be the number of measurements of value $d_{i}$. Let $N$ be the total number of measurements to the same pair of sensors. We have $N=\sum_{i=1}^{n} k_{i}$. Based on the distribution of the measured values, we observe two phenomena that are helpful in identifying correct distance measurements. First, a distance between a pair of sensors tends to have a larger $N$ if it is less affected by obstructions. This is because fewer distance measurements are observed by beacons when the pair of sensors are surrounded by obstructions and therefore are difficult for the virtual ruler to access. Second, among all the distance values measured to the same pair of sensors, the value with the largest number of measurements $k_{\max }$ has the highest probability to be the correct distance measurement. Because a typical indoor environment contains more open spaces than obstructions, pairwise sensors have higher probability of being observed by mobile beacons through line-ofsight paths than through reflected paths. Based on the observations above, we choose the value with the largest number of measurements $k_{\max }$ as the correct distance measurement between a pair of sensors. Moreover, we assign confidence $C$ to a distance measurement according to $N$ and $k_{\max }$ as $C=N+\lambda \times k_{\max }$, where $\lambda$ is the weighting coefficient. Based on the confidence of distance measurements, we combine the virtual ruler distance measurement with the recursive approach, in which the distance measurements with higher confidence will have higher priorities to be applied.

\section{E. Combine the virtual ruler distance measurement with the recursive approach}

In the recursive approach, a few sensors are pointed as beacons whose positions are determined through out-of- 


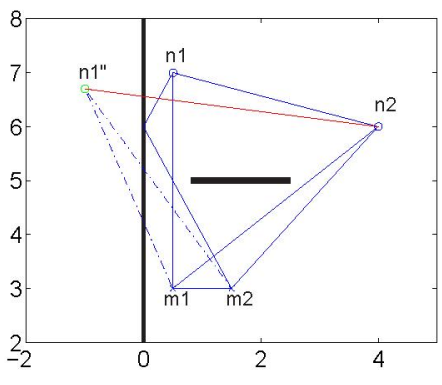

(a) example a

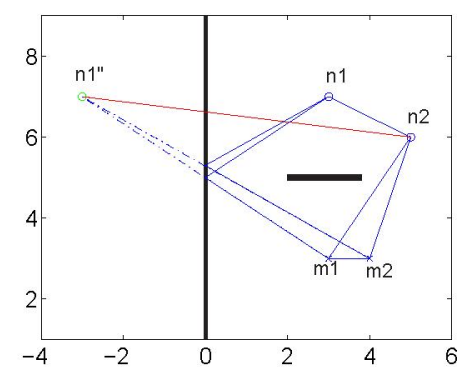

(b) example b

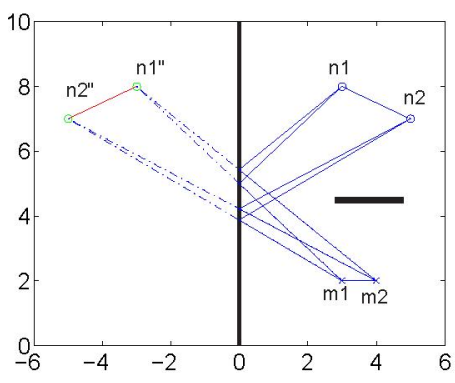

(c) example c

Fig. 8 Examples of distances estimated by mobile beacons in an obstructed environment

band approaches such as manual measurements. In order to distinguish the beacons used by recursive approaches from the mobile beacons used by distance measurement, we define the former as the static beacons. Recursive approaches first locate sensors that are close to static beacons and iteratively convert sensors to beacons after their positions are determined. Consequently, the static beacons can be propagated from the initial beacons to an entire deployed area such that all sensors can be localized. In the process of the recursive approaches, multiple candidates are often available to be converted to new beacons. Distance measurements between pairwise sensors are also redundant in a densely deployed sensor network such that sensors can be localized by using only partial distance measurements. The localization result will not be affected by the sequence of converting sensors to beacons and the distance measurement subset if all the distance measurements have the same error distribution. However, in an obstructed indoor environment, where correct distances are mixed together with incorrect distances, it is critical to choose the optimal sequence of the recursive process such that the incorrect distance measurement can be excluded from the distance measurement subset used in the recursive process. Based on the confidence assigned to distance measurement by our mobile beacons, the optimal recursive sequence can be approached as follows. In each step of the recursive approach, the candidate is selected such that we can maximize the confidence of all the distance measurements that are used to locate the candidate.

\section{F. Moving strategy}

We assume that the virtual ruler moves around as below. It follows a step by step movement pattern. For each moving step, the virtual ruler randomly selects a moving direction. However, our test shows that such a random moving strategy leads to a non-uniform distribution of the moving tracks. As shown in Fig. 9, the virtual ruler only visits the right part of the floor while ignoring the left part. To improve the coverage of the virtual ruler, we assume that the virtual ruler has certain intelligence such that they can communicate with nearby sensors to decide the moving direction. During the process of measurement, each sensor keeps recording how many times it has been measured. The virtual ruler queries neighboring sensors before it makes the moving decision. Based on the queried results, the virtual ruler moves towards the sensor that have least measurement times. The moving track of the enhanced moving strategy is shown in Fig. 10, where the floor is uniformly covered by the virtual ruler and all the sensors are visited.

\section{Performance evaluation}

We evaluate our virtual ruler approach in a $20 \mathrm{~m} \times 20 \mathrm{~m}$ square area where 50 sensors are randomly deployed. Two configurations of the deployed area are used in our simulation: i) 20 obstructions are randomly positioned (Fig. 11); ii) the square area is configured into a real indoor environment where rooms are separated from each other by walls (Fig. 10). The virtual ruler moves around in the area and measures pairwise distances of static sensors within their measurable range. As illustrated in Fig. 10, the dashed lines show the trace of the virtual ruler that moves randomly throughout the region. The virtual ruler moves a total of 100 steps, and stops at each step to perform measurements. In this section, we evaluate both the distance measurement performance and the localization performance when combining the virtual ruler approach with the recursive approach.

\section{A. Distance measurement performance of the mobile beacon approach}

When the virtual ruler moves around in the deployed area, it can measure the distance between a pair of 


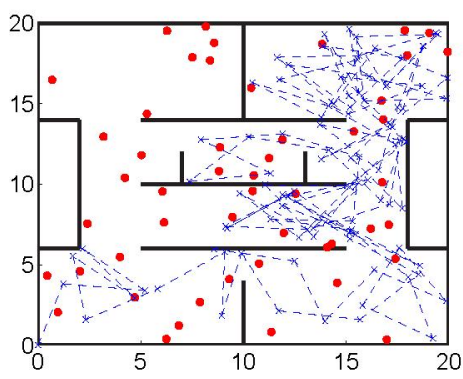

Fig. 9 Beacons' moving tracks with random moving strategy

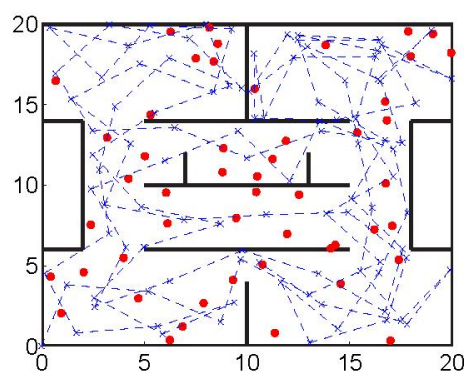

Fig. 10 Beacons' moving tracks with enhanced moving strategy

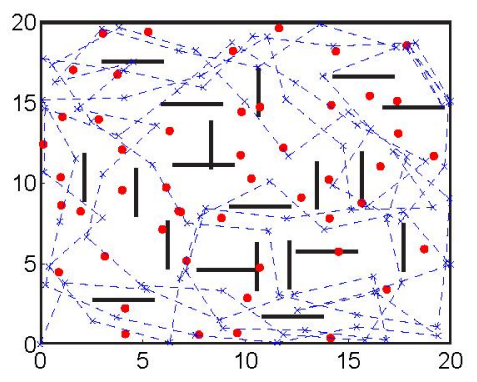

Fig. 11 Sensors deployment with randomly distributed obstructions sensors multiple times from different perspectives. For the distance between the same pair of sensors, the virtual ruler may obtain different values, among which the correct value is mixed together with the incorrect ones. In order to statistically identify the correct value from the incorrect ones, we investigate the characteristics of the distance measurement distribution through intensive simulations conducted in indoor environments. We record all the distance measurement values obtained by the virtual ruler and plot the distribution of measured values in Fig. 12, where Fig. 12(a) shows the full distribution of all the distance measurements and Fig. 12(b) shows an enlarged part of Fig. 12(a) for clear visualization. The height of each vertical bar represents the total number of distance measurements $N$ between a pair of sensors. The vertical bar is further divided into two segments and the height of the the bottom segment represents the number of measurements $k_{\max }$ of the value $d_{\max }$, which has the largest number of measurements among all the values observed by the virtual ruler. The sum of the number of measurements of all other values is represented as the length of the top bar. The bottom bar is colored in gray if it is the correct value; otherwise the bar is colored in black. Fig. 12 shows that the majority of bottom bars are painted in gray, which means the correct distance measurements tend to be observed by the virtual ruler more frequently than incorrect ones. Therefore, among all the measured distance values to the same pair of sensors, we can select the one with the highest observed frequency as the correct distance measurement.

We compare the distance measurement of the mobileassisted approach [20], virtual ruler approach, virtual ruler approach with frequency analysis in both the indoor environment and random obstruction environment. The comparison results are shown in Table II and Table III respectively. The comparison shows that the virtual ruler approach has lower percentage of incorrect distance mea- surement than the mobile-assisted approach. Moreover, the percentage of incorrect distance measurements of the virtual ruler approach can be significantly reduced by frequency analysis.

From Fig. 12, we can find a few counter examples where incorrect distance measurements fall to the bottom bars that are painted in black. However, the bar that contains the correct distance measurement at the bottom tends to be high. This observation motivates us to further exclude incorrect distance measurement by imposing a threshold to distance measurement. When the total number of distance measurements falls below the threshold, we regard the distance measurements as incorrect ones. By using the threshold strategy, the percentage of incorrect distance measurement is further reduced, as shown in Table II and III.

B. localization performance by combining the mobile beacons distance measurement with recursive approaches

The observations above shows that a distance measurement can be evaluated through two metrics: i) the total number of measurements to the same distance between pairwise sensors; ii) among all the measurements, the proportion of the value that has the largest contribution to the total number of measurements. Based on the two metrics, a confidence value can be assigned to each distance measurement such that we can rank distance measurements according to their confidences. With the ranked distance measurements, the virtual ruler distance measurement can be readily combined with the recursive approach to exclude incorrect distance measurements by giving a higher priority to a distance measurement with higher confidence. We then evaluate the combination of the virtual ruler approach and the recursive approach in the indoor floor environment. Besides the distance measurement errors incurred by the obstructions, we add 


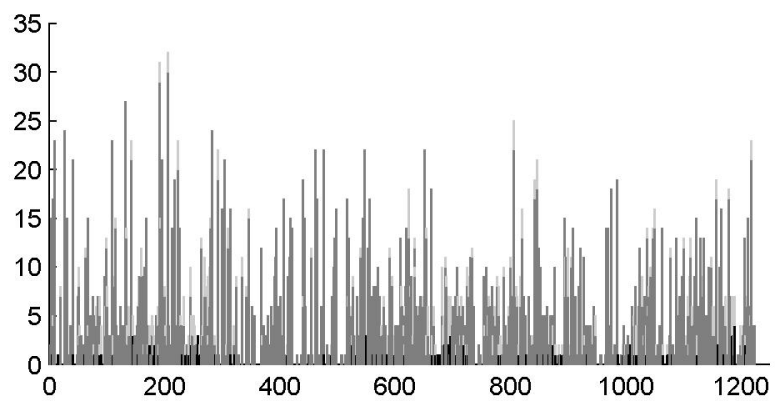

(a) Full distribution

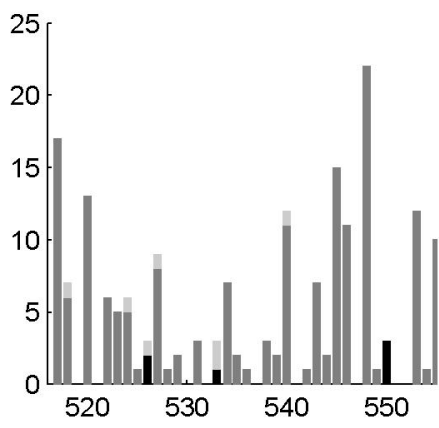

(b) Enlarged part

Fig. 12 Distance measurement distribution

TABLE II Distance measurement comparison in an indoor floor environment

\begin{tabular}{|l|l|l|l|l|}
\hline distance measurement approach & $\begin{array}{l}\text { mobile- } \\
\text { assisted }\end{array}$ & $\begin{array}{l}\text { virtual ruler with } \\
\text { one time measure- } \\
\text { ment }\end{array}$ & $\begin{array}{l}\text { virtual ruler with virtual ruler with } \\
\text { frequency analysis } \\
\text { threshold strategy }\end{array}$ \\
\hline total number of measurements & 486 & 658 & 658 & 201 \\
\hline the number of incorrect measurements & 129 & 146 & 100 & 2 \\
\hline percentage of incorrect measurements & $26.54 \%$ & $22.19 \%$ & $15.2 \%$ & $1 \%$ \\
\hline
\end{tabular}

TABLE III Distance measurement comparison in an area with randomly distributed obstructions

\begin{tabular}{|l|l|l|l|l|}
\hline distance measurement approach & $\begin{array}{l}\text { mobile- } \\
\text { assisted }\end{array}$ & $\begin{array}{l}\text { virtual ruler with } \\
\text { one time measure- } \\
\text { ment }\end{array}$ & $\begin{array}{l}\text { virtual ruler with } \\
\text { frequency analysis }\end{array}$ & $\begin{array}{l}\text { virtual ruler with } \\
\text { threshold strategy }\end{array}$ \\
\hline total number of measurements & 579 & 740 & 740 & 253 \\
\hline the number of incorrect measurements & 159 & 166 & 104 & 3 \\
\hline percentage of incorrect measurements & $27.46 \%$ & $22.43 \%$ & $14.05 \%$ & $1.2 \%$ \\
\hline
\end{tabular}

Gaussian distributed random error to distance measurement. The simulation result is shown in Fig. 13 and Fig. 14. Fig. 13 shows that the virtual ruler successfully excludes most incorrect distance measurements and only two incorrect ones (painted with two bold dashed lines) are involved in the localization. In Fig. 14, the circles represent the true positions of sensors and lines represent the localization errors between true positions and the estimated positions.

We further compare the virtual ruler approach with the iterative least squares fitting algorithm in [21] that filters out incorrect distance measurements by iteratively applying least squares fitting. In each iteration the estimated distances between a sensor and static beacons are calculated based on the sensor's position estimated by least squares fitting. The distance measurement that differs most from its estimated value is excluded in the next iteration. Fig. 15 shows the comparison result in the indoor environment. We can see that the virtual ruler distance measurement combined with the recursive approach outperforms the iterative least squares fitting algorithm. The iterative least squares fitting algorithm fits all the measured distances equally such that the final estimated location is the averaged result of all the measurement. However, the least squares fitting algorithm does not guarantee that the estimated result will favor the correct distance measurements. If the estimated location favors the incorrect distance measurements, the correct distance measurement will be filtered in the iteration.

\section{CONClusion}

It is a challenging task to locate sensors in an indoor environment because the multipath effects will incur large errors in distance measurements. The incorrect distance measurements, once mixed together with correct distance measurements, are difficult for localization algorithms to identify and exclude. In this paper, we proposed to filter out the incorrect distance measurement in the 


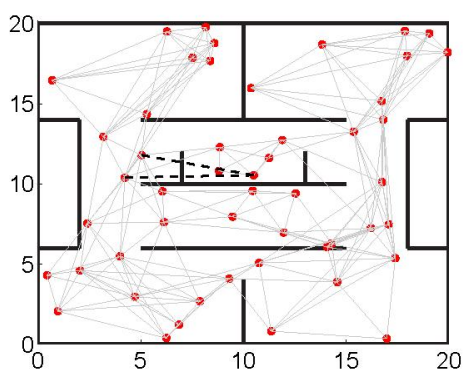

Fig. 13 Distance measurement subset selected by virtual ruler

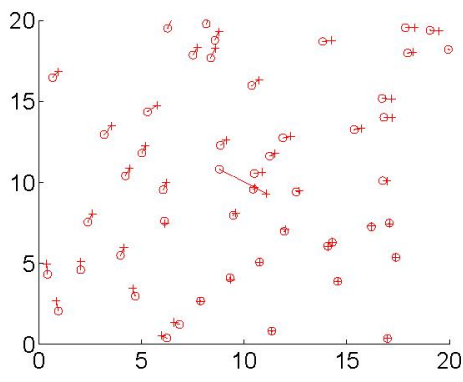

Fig. 14 applying virtual ruler to the recursive approach

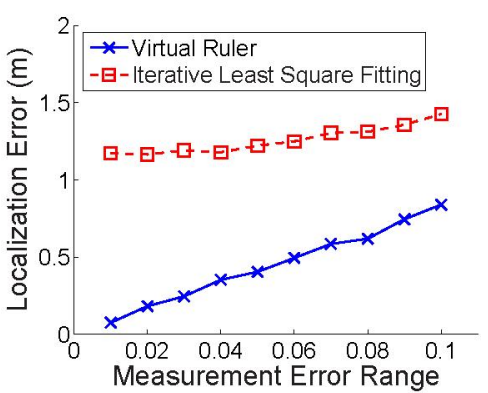

Fig. 15 Compare virtual ruler and iterative least squares fitting first step of distance measurement. By using mobile beacons to measure distances between pairwise sensors from multiple perspectives, our proposed mobile beacon based distance measurement can statistically identify incorrect distance measurements, which provides a good basis for indoor localization algorithms. Especially, the mobile beacon based distance measurement can be further combined with the recursive approach such that distance measurements with higher confidence are selected with higher priority. The performance evaluation shows that the mobile beacon based distance measurement can achieve better localization results than previous mobileassisted approaches.

\section{ACKNOWLEDGMENT}

This work was supported in part by the US National Science Foundation under grants CCF-0325760, CCF0514078 , CNS-0549006, and CNS 0551464.

\section{REFERENCES}

[1] J. Aslam, Z. Butler, F. Constantin, V. Crespi, G. Cybenko, and D. Rus, "Tracking a moving object with a binary sensor network," in ACM SenSys, 2003

[2] A. Mainwaring, J. Polastre, R. Szewczyk, and D. Culler, "Wireless Sensor Networks for Habitat Monitoring," in WSNA, 2002.

[3] G. Simon, M. Marti, A. Ldeczi, G. Balogh, B. Kusy, A. Ndas, G. Pap, J. Sallai, and K. Frampton, "Sensor Network-Based Countersniper System," in SenSys, 2004.

[4] B. Karp and H. T. Kung, "GPSR: Greedy Perimeter Stateless Routing for Wireless Networks," in MobiCom, 2000.

[5] Y. Xu, J. Heidemann, and D. Estrin, "Geography-informed Energy Conservation for Ad Hoc Routing," in MobiCom, 2001.

[6] J. Hightower, R. Want, and G. Borriello, "SpotON: An Indoor 3D Location Sensing Technology Based on RF Signal Strength," UW CSE 00-02-02, University of Washington, Department of Computer Science and Engineering, Seattle, WA, 2000 .

[7] K. Whitehouse, "The design of calamari: an ad-hoc localization system for sensor networks," Master's Thesis, University of California at Berkeley, 2002.

[8] N. Bulusu, J. Heidemann, and D. Estrin, "Adaptive Beacon Placement," in ICDCS, 2001.
[9] T. He, C. Huang, B. M. Blum, J. A. Stankovic, and T. F Abdelzaher, "Range-Free Localization Schemes in Large Scale Sensor Networks," in MobiCom, 2003.

[10] D. Niculescu and B. Nath, "Ad hoc Positioning System (APS)," in GLOBECOM, 2001.

[11] A. Savvides, H. Park, and M. B. Srivastava, "The n-hop multilateration primitive for node localization problems," in Mobile Networks and Applications, 2003.

[12] Y. Shang, W. Ruml, Y. Zhang, and M. P. J. Fromherz, "Localization from mere connectivity," in MobiHoc, 2003.

[13] L. Girod and D. Estrin, "Robust Range Estimation Using Acoustic and Multimodal Sensing," in IROS, 2001.

[14] A. Savvides, C. Han, and M. B. Strivastava, "Dynamic finegrained localization in ad-hoc networks of sensors," in MobiCom, 2001

[15] J. Albowicz, A. Chen, and L. Zhang, "Recursive Position Estimation in Sensor Networks," in ICNP, 2001.

[16] D. Moore, J. Leonard, D. Rus, and S. Teller, "Robust Distributed Network Localization with Noisy Range Measurements," in SenSys, 2004.

[17] W. Zhao, M. Ammar, and E. Zegura, "A message ferrying approach for data delivery in sparse mobile ad hoc networks," in MobiHoc, 2004.

[18] M. M. B. Tariq, M. Ammar, and E. Zegura, "Message ferry route design for sparse ad hoc networks with mobile nodes," in MobiHoc, 2006.

[19] M. Sichitiu and V. Ramadurai, "Localization of wireless sensor networks with a mobile beacon," in MASS, 2004.

[20] N. B. Priyantha, H. Balakrishnan, E. Demaine, and S. Teller, "Mobile-assisted localization in wireless sensor networks," in INFOCOM, 2005.

[21] M. Kushwaha, K. Molnar, J. Sallai, P. Volgyesi, M. Maroti, and A. Ledeczi, "Sensor node localization using mobile acoustic beacons," in MASS, 2005.

[22] K. Whitehouse, C. Karlof, A. Woo, F. Jiang, and D. Culler, "The effects of ranging noise on multihop localization: an empirical study," in IPSN, 2005.

[23] K. Whitehouse and D. Culler, "Calibration as parameter estimation in sensor networks," in WSNA, 2002.

[24] A. Smith, H. Balakrishnan, M. Goraczko, and N. Priyantha, "Tracking moving devices with the cricket location system," in MobiSys, 2004

[25] J. Hill and D. Culler, "Mica: A wireless platform for deeply embedded networks," IEEE Micro, 2002. 